\title{
THE MACKEY CONTINUITY OF THE MONOTONE REARRANGEMENT
}

\author{
ANTHONY HORSLEY AND ANDRZEJ J. WROBEL
}

\begin{abstract}
Let $(A, A, \mu)$ be a probability space, and let mes denote the Lebesgue measure on the Borel $\sigma$-algebra $B$ in $[0,1]$. The nondecreasingrearrangement operator from the space $L^{\infty}(\mu)=L^{\infty}(A, \mathcal{A}, \mu)$ of real-valued essentially bounded functions into $L^{\infty}=L^{\infty}([0,1], B$, mes) is shown to be uniformly continuous in the Mackey topologies $\tau\left(L^{\infty}(\mu), L^{1}(\mu)\right)$ and $\tau\left(L^{\infty}, L^{1}\right)$ on $L^{\infty}(\mu)$ and $L^{\infty}$, respectively.
\end{abstract}

1. Definitions and notation. Let $(A, \mathcal{A}, \mu)$ be a probability space and let mes denote the Lebesgue measure on the $\sigma$-algebra $B$ of Borel subsets of $[0,1]$. For any real-valued $A$-measurable function $f$ on $A$ there is a unique, right-continuous, nondecreasing function $f_{\uparrow}$ on $[0,1]$ such that $\operatorname{mes}\left(f_{\uparrow}\right)^{-1}=\mu f^{-1}$, i.e., $f_{\uparrow}$ and $f$ are equidistributed. The function $f_{\uparrow}$ is called the nondecreasing rearrangement of $f$. The nonincreasing rearrangement $f_{\downarrow}$ of $f$ is defined as $f_{\downarrow}=-(-f)_{\uparrow}$, cf. [3, p. 932]. For any $1 \leq p \leq+\infty$, if $f \in L^{p}(\mu)=L^{p}(A, A, \mu)$, then $f_{\uparrow} \in L^{p}=L^{p}([0,1], B$, mes $)$. The term "rearrangement operator" is always used to mean the nondecreasingrearrangement operator $\uparrow$.

The Hardy-Littlewood-Pólya spectral order $\prec$ is defined as follows: $f \prec g$ if and only if $\int_{t}^{1} f_{\uparrow} d$ mes $\leq \int_{t}^{1} g_{\uparrow} d$ mes for all $t \in[0,1]$ with equality for $t=0$.

For a pair of vector spaces $E$ and $F$ in duality, let $\sigma(E, F)$ and $\tau(E, F)$ denote, respectively, the weak and Mackey topologies on $E$ induced by $F$. The duality between $L^{p}(\mu)$ and $L^{p^{\prime}}(\mu)$, where $p^{-1}+\left(p^{\prime}\right)^{-1}=1$, is $\langle f, g\rangle_{\mu}=\int_{A} f g d \mu$, and the subscript $\mu$ is suppressed when $\mu=$ mes.

2. The Mackey continuity of the rearrangement operator. For $1 \leq$ $p<+\infty$, the rearrangement operator $\uparrow$ is a nonexpansive mapping of $L^{p}(\mu)$ into $L^{p}$ (with their usual norms). This result is contained in [3, Corollary (5.2)] with $\phi(x, y)=-|x-y|^{p}$; note also [3, Remark (5.7)]. Alternatively, it can be seen from the relation $f_{\uparrow}-g_{\uparrow} \prec f-g[3,(6.1)$ (ii)], which, in view of the monotonicity of the $L^{p}$-norm in the preorder $\prec\left[\mathbf{2}\right.$, Theorem (2.5)], implies $\left\|f_{\uparrow}-g_{\uparrow}\right\|_{p} \leq\|f-g\|_{p}$. Note also that for $p=2$ the nonexpansive property of the operator $\uparrow$ follows directly from the Hardy-Littlewood-Pólya inequality $\langle f, g\rangle_{\mu} \leq\left\langle f_{\uparrow}, g_{\uparrow}\right\rangle$.

For $p=+\infty$, the rearrangement operator is nonexpansive in the essential supremum norm. For an economic application in [6], however, it is necessary to establish the continuity of the rearrangement operator in some topologies on $L^{\infty}(\mu)$ and $L^{\infty}$ in which the respective continuous duals are $L^{1}(\mu)$ and $L^{1}$. From the following example it is seen that the rearrangment operator on $L^{\infty}$ into $L^{\infty}$ is discontinuous in the weak topology $\sigma\left(L^{\infty}, L^{1}\right)$.

Received by the editors June 17, 1985.

1980 Mathematics Subject Classification. Primary 46E30; Secondary 47H09.

Key words and phrases. Nondecreasing rearrangement, Mackey topology. 
(2.1) EXAMPLE. Let $f^{n}(t)=\operatorname{sgn} \sin 2 \pi n t$ for $t \in[0,1]$ and $n=1,2, \ldots$ One has $f^{n} \rightarrow 0$ in $\sigma\left(L^{\infty}, L^{1}\right)$; but for all $n, f^{n}$ is equal to $(-1)$ on $[0,1 / 2)$ and to +1 on $[1 / 2,1]$.

As this example suggests, continuity holds for the Mackey topology.

(2.2) THEOREM. The rearrangement operator $\uparrow$ is a uniformly continuous mapping of $L^{\infty}(\mu)$ endowed with the Mackey topology $\tau\left(L^{\infty}(\mu), L^{1}(\mu)\right)$ into $L^{\infty}$ endowed with the Mackey topology $\tau\left(L^{\infty}, L^{1}\right)$.

Proof. The Mackey topology $\tau(E, F)$ is the topology of uniform convergence on $\sigma(F, E)$-compact sets (see, for example, [9, Corollary 1 to Theorem IV.3.2, and subsequent remarks]). By the Dunford-Pettis compactness criterion, weak relative compactness is equivalent to uniform integrability (see [5, Theorems IV.8.9 and V.6.1]).

Take any uniformly integrable $\Phi \subset L^{1}$. To prove the Theorem, it is necessary and sufficient to find a uniformly integrable $\Psi \subset L^{1}(\mu)$ such that, for any $f, g \in L^{\infty}(\mu)$, if $\left|\langle\psi, g-f\rangle_{\mu}\right|<1$ for all $\psi \in \Psi$, then $\left|\left\langle\phi, g_{\uparrow}-f_{\uparrow}\right\rangle\right|<1$ for all $\phi \in \Phi$.

From $[3,(6.1)(\mathrm{ii})]$

$$
g_{\uparrow}-f_{\uparrow} \prec g-f \quad \text { and } \quad f_{\uparrow}-g_{\uparrow} \prec f-g .
$$

By the Hardy-Littlewood-Pólya inequality (see, for example, [4, Lemma (3.4)]), (2.3) implies

$$
\left\langle\phi, g_{\uparrow}-f_{\uparrow}\right\rangle \leq\left\langle\phi_{\uparrow},(g-f)_{\uparrow}\right\rangle \quad \text { for all } \phi \in L^{1}
$$

and

$$
\left\langle\phi, f_{\uparrow}-g_{\uparrow}\right\rangle \leq\left\langle\phi_{\uparrow},(f-g)_{\uparrow}\right\rangle \quad \text { for all } \phi \in L^{1} .
$$

This last inequality can also be written equivalently as

$$
\left\langle\phi, g_{\uparrow}-f_{\uparrow}\right\rangle \geq\left\langle\phi_{\downarrow},(g-f)_{\uparrow}\right\rangle \text { for all } \phi \in L^{1} .
$$

Consider first the case of a nonatomic $\mu$. Let $S$ be the set of all measurepreserving mappings of $(A, A, \mu)$ into $[0,1]$. By [4, Proposition (3.3) or 8, Lemma $1]$, for any $f$ and $g$ there exists $S \in S$ such that $g-f=(g-f)_{\uparrow} \circ S$. Then for such $S$,

$$
\left\langle h,(g-f)_{\uparrow}\right\rangle=\langle h \circ S, g-f\rangle_{\mu} \quad \text { for all } h \in L^{1} .
$$

Using (2.6) for $h=\phi_{\uparrow}, \phi_{\downarrow}$ with (2.4) and (2.5) gives

$$
\inf _{S \in S}\left\langle\phi_{\downarrow} \circ S, g-f\right\rangle_{\mu} \leq\left\langle\phi, g_{\uparrow}-f_{\uparrow}\right\rangle \leq \sup _{S \in S}\left\langle\phi_{\uparrow} \circ S, g-f\right\rangle_{\mu} \quad \text { for all } \phi \in L^{1} .
$$

Consider the set $\Phi_{\uparrow} \circ S=\left\{\phi_{\uparrow} \circ S \mid \phi \in \Phi, S \in S\right\}$ : It is a uniformly integrable subset of $L^{1}(\mu)$ because uniform integrability is a property which depends only on the distributions of the functions involved. It follows from (2.7) that it suffices to take $\Psi=\Phi_{\uparrow} \circ S$. This completes the proof for a nonatomic $\mu$.

Only a slight modification of the preceding argument has to be made for a $\mu$ with atoms. By [7, Theorem (7.1) or 3, p. 390, first line], there exist $\bar{\phi}, \phi \in L^{1}(\mu)$ such that

$$
\left\langle\phi_{\uparrow},(g-f)_{\uparrow}\right\rangle=\langle\bar{\phi}, g-f\rangle_{\mu} \quad \text { and } \quad \bar{\phi} \prec \phi
$$


and

$$
\left\langle\phi_{\downarrow},(g-f)_{\uparrow}\right\rangle=\langle\underline{\phi}, g-f\rangle_{\mu} \quad \text { and } \quad \underline{\phi} \prec \phi .
$$

Let $\Omega(\phi)=\left\{\psi \in L^{1}(\mu) \mid \psi \prec \phi\right\}$ and $\Omega(\Phi)=\bigcup_{\phi \in \Phi} \Omega(\phi)$. By [1, Corollary 4.3 and [7, Theorem 9.5 , the set $\Omega(\Phi)$ is uniformly integrable because $\Phi$ is. From (2.4), (2.5), (2.8) and (2.9) it follows that

$$
\begin{aligned}
\inf _{\psi \in \Omega(\Phi)}\langle\psi, g-f\rangle_{\mu} & \leq\left\langle\phi, g_{\uparrow}-f_{\uparrow}\right\rangle \\
& \leq \sup _{\psi \in \Omega(\Phi)}\langle\psi, g-f\rangle_{\mu} \quad \text { for all } \phi \in \Phi .
\end{aligned}
$$

It follows from (2.10) that it suffices to take $\Psi=\Omega(\Phi)$. Q.E.D.

(2.11) REMARK. For countable sequences, convergence in the Mackey topology $\tau\left(L^{\infty}(\mu), L^{1}(\mu)\right)$ is equivalent to convergence in the measure $\mu$ and boundedness in the essential supremum norm. Therefore, sequential continuity of the rearrangement operator in the Mackey topologies under consideration follows also from its continuity in the topologies of convergence in the measures $\mu$ and mes.

(2.12) REMARK. Even after their restriction to the range of the operator $\uparrow$, viz., the cone of nondecreasing functions in $L^{\infty}$, the Mackey topology $\tau\left(L^{\infty}, L^{1}\right)$ is strictly stronger than the weak topology $\sigma\left(L^{\infty}, L^{1}\right)$.

\section{REFERENCES}

1. K. M. Chong, Spectral orders, uniform integrability and Lebesgue's dominated convergence theorem, Trans. Amer. Math. Soc. 191 (1974), 395-404.

2. Some extensions of a theorem of Hardy, Littlewood and Pólya and their applications, Canad. J. Math. 26 (1974), 1321-1340.

3. P. W. Day, Rearrangement inequalities, Canad. J. Math. 24 (1972), 930-943.

4. - Decreasing rearrangements and doubly stochastic operators, Trans. Amer. Math. Soc. 178 (1973), 383-392.

5. N. Dunford and J. T. Schwartz, Linear operators. Part I: General theory, Pure and Appl. Math., vol. 7, Interscience, New York, 1958.

6. A. Horsley and A. Wrobel, The formal theory of pricing and investment for electricity. I: A continuous-time model of deterministic production, ICERD Discussion Paper No. 86/135, London School of Economics.

7. W. A. J. Luxemburg, Rearrangement invariant Banach function spaces, Queen's Papers in Pure and Appl. Math. 10 (1967), 83-144.

8. J. V. Ryff, Orbits of $L^{1}$-functions under doubly stochastic transformations, Trans. Amer. Math. Soc. 117 (1965), 92-100.

9. H. H. Schaefer, Topological vector spaces, Graduate Texts in Math., vol. 3, Springer-Verlag, Berlin and New York, 1971.

DEPARTMENT OF ECONOMICS, LONDON SCHOOL OF ECONOMICS AND POLITICAL SCIENCE, UNIVERSity OF LONDON, HOUghtoN STREET, LONDON WC2A 2AE, UNited KINGDOM 